\title{
Internet-Based Methods May Reach Higher-Risk Men who have Sex with Men Not Reached Through Venue-Based Sampling ${ }^{\S}$
}

\author{
Travis Sanchez ${ }^{*}$, Amanda Smith, Damian Denson, Elizabeth DiNenno and Amy Lansky, \\ for the Web-Based HIV Behavioral Surveillance Study Group
}

Centers for Disease Control and Prevention, Division of HIV/AIDS Prevention, Atlanta, GA, USA

\begin{abstract}
Background: Internet-based sampling methods may reach men who have sex with men (MSM) who don't attend physical venues frequented by MSM and may be at higher risk of HIV infection.

Methods: Multivariate logistic regression was used to examine characteristics of adult MSM participants in 2 studies conducted in the same 5 U.S. cities: the 2003-2005 National HIV Behavioral Surveillance System (NHBS) which used sampling from physical MSM venues (e.g., bars, clubs) and the 2007 Web-based HIV Behavioral Surveillance (WHBS) pilot which used sampling through online banner advertisements.

Results: Among 5024 WHBS MSM, 95\% attended a physical MSM venue in the past 12 months, and 75\% attended weekly. WHBS MSM who were black, aged 18-21 years, not college educated, bisexual- or heterosexual-identifying, and reported unknown HIV serostatus were less likely to have attended a physical MSM venue in the past 12 months (all $\mathrm{p}<0.01)$. Compared to NHBS MSM, WHBS MSM were more likely to be white, younger, college-educated, report unknown HIV serostatus, report unprotected anal intercourse with a casual partner, and have first met that partner online (all $\mathrm{p}<0.0001)$. WHBS MSM were less likely to have been under the influence of drugs during most recent $\operatorname{sex}(\mathrm{p}=0.01)$ or not know their sex partner's HIV serostatus $(\mathrm{p}<0.0001)$.

Conclusions: Many MSM recruited online also attended physical venues, but attendance varied by sub-group. Participants in WHBS and NHBS differed, and WHBS may represent a group of MSM at higher risk of HIV infection. These findings suggest that an internet-based method may be a useful supplement to NHBS.
\end{abstract}

Keywords: MSM, HIV, internet, gay, sex.

\section{INTRODUCTION}

Men who have sex with men (MSM) have always been the most affected risk group in the US HIV epidemic, and recent evidence confirms that MSM in the US and in other industrialized countries are experiencing a resurgence in HIV transmission since at least $2000[1,2]$. The Centers for Disease Control and Prevention (CDC) released the HIV Prevention Strategic Plan in 2000 with a goal to reduce new HIV infections in the US. Objectives to reach this goal included strengthening national capacity to monitor the HIV epidemic to better direct and evaluate prevention efforts [3]. In an effort to meet this objective, $\mathrm{CDC}$ awarded funds to state and local health departments to develop and implement a new surveillance system, the National HIV Behavioral Surveillance System (NHBS). NHBS is an ongoing surveillance system to monitor trends in HIV-related risk behaviors [4]. From 2003-2005, NHBS focused on data collection among MSM in 17 metropolitan statistical areas (MSAs) in the U.S. [5].

\footnotetext{
*Address correspondence to this author at the Emory University, 1518 Clifton Road NE, Atlanta, GA 30322, USA; Tel: +1 404727 8403;

Fax: +1 404727 8737; E-mail: Travis.Sanchez@emory.edu

${ }^{8}$ The findings and conclusions in this report are those of the authors and do not necessarily represent the views of the U.S. Centers for Disease Control and Prevention.
}

The sampling method for NHBS among MSM (NHBSMSM) uses physical venues, such as bars, clubs or other locations, where MSM are approached and recruited for an in-person interview [6]. NHBS investigators hypothesized that some MSM of interest for behavioral surveillance may not be reached through this sampling method. Past studies specific to MSM suggest that internet usage rates for this group are higher than for other men, and approximately $40 \%$ of MSM have reported seeking sex partners on the internet [7-10]. Some research has also identified high rates of sexual risk behavior among MSM who meet sexual partners on the internet. Sexual risk behavior may be more common among these MSM than those who meet partners elsewhere [10], and internet use to find sex partners has been implicated in the transmission of sexually transmitted diseases (STDs) [11-13].

The Web-based HIV Behavioral Surveillance System (WHBS) pilot project was initiated in 2005 to examine whether the internet may be used to collect behavioral data from MSM populations that may not be sampled through NHBS. Internet-based behavioral research is an emerging field, but several researchers have successfully implemented online behavioral research projects using a variety of convenience sampling methods, some of which have focused on HIV and MSM [14-18]. Another report described the details of the WHBS methodology and showed that implementation of an internet-based systematic sampling 
method of MSM may be problematic, but a convenience sample of MSM using banner ads is feasible and produced useful and timely behavioral information from a large number of MSM [19]. The purpose of this report is to investigate whether WHBS accessed a group of MSM not otherwise reachable through NHBS sampling methods and determine whether MSM recruited through WHBS were more likely to report HIV-related risk behavior than MSM recruited through NHBS.

\section{METHODS}

\section{Web-Based HIV Behavioral Surveillance System (WHBS)}

WHBS was an internet-based survey conducted in 2007 to measure HIV-related risk and preventive behaviors of MSM who reported currently residing in the following metropolitan statistical areas (MSAs): Baltimore, Maryland; Boston, Massachusetts; Dallas, Texas; Los Angeles, California; New York, New York; and San Francisco, California. WHBS data from Dallas are not included in this report because there was no comparable NHBS data from this MSA for 2003-2005. Details of the methods for the WHBS pilot project are reported elsewhere [19]. Briefly, banner advertisements (banner ads) were used to recruit persons who accessed MSM-related websites (e.g., Gay.com, Manhunt.net, Dlist) and social networking websites (e.g., MySpace and Friendster). Banner ads briefly explained the purpose of the survey, encouraged men to participate, and provided a link to the WHBS website. When the users clicked on the link, they were assigned a unique survey identification number (ID) and sent directly to the WHBS eligibility screening webpage.

WHBS eligibility criteria included being age $\geq 18$ years, male at birth, and a current resident of a participating MSA. Eligible persons were sent to a consent webpage and were asked to check a box stating that they had read the information about WHBS and agreed to participate in a 10to 15-minute anonymous web-based survey without reimbursement. Participants could view and make corrections to any of their previous answers, but after they exited the WHBS website they were unable to return to any of their previous survey responses. The WHBS survey consisted of several question domains: demographics; venue attendance and internet usage; sexual and drug use behaviors; HIV and STD testing experiences; and utilization of HIV prevention services.

\section{National HIV Behavioral Surveillance System (NHBS)}

NHBS data used for this analysis were collected from MSM during 2003 through 2005 in the same 5 MSAs from which WHBS recruited. Eligibility criteria for NHBS-MSM included being age $\geq 18$ years, male at birth, a current resident of a participating MSA, and not having previously participated in NHBS-MSM during the current cycle.

Details of the NHBS-MSM sampling and data collection methods have been previously reported $[5,6]$. The main steps are as follows: venues frequented by MSM (e.g., bars, dance clubs, social organizations, restaurants, retail businesses) were identified, optimal days of the week and times of day for sampling at each venue were determined, and then sampling events were chosen using random selection methods. Men were systematically recruited at venues during sampling events. Trained interviewers administered a 20-minute standardized questionnaire with survey domains and questions comparable to those described for WHBS.

\section{Measures}

To answer the question of whether WHBS reached MSM not potentially reached through NHBS-MSM methods, we examined WHBS MSM attendance at the types of public venues which were used for NHBS-MSM sampling. Attendance was measured as the average frequency of venue attendance during the past 12 months ("Did not attend", "Attended monthly or less", and "Attended weekly or more") and attendance was also stratified by venue type. We also measured the average frequency of NHBS MSM use of gay or bisexual internet chatrooms during the past 12 months.

To determine the extent to which we obtained a different sample of MSM using WHBS methods than NHBS-MSM methods, we examined participant characteristics and HIVrelated risk behaviors. The following characteristics and behaviors were compared between the NHBS-MSM and WHBS samples: race/ethnicity, age, college education, sexual identity, self-reported HIV serostatus, unprotected anal intercourse during last sex with a male partner, first meeting the most recent male sex partner online, being under the influence of drugs during last sex with a male partner, and not knowing the HIV serostatus of the most recent male sex partner. To conduct these comparisons, we had to create subsets of data from NHBS-MSM and WHBS so that the measures were comparable. NHBS-MSM only collected information about where a male sex partner was first met if that partner was a casual partner. A casual partner in both studies was defined as "someone you do not feel committed to above anyone else, or someone who is not a boyfriend, spouse, significant other, or life partner." WHBS only collected detailed information about sexual behavior about the "last time you had anal or oral sex with your most recent [male] partner." Both NHBS-MSM and WHBS only collected knowledge of HIV serostatus of the most recent male sex partner defined by response to the question, "The last time you had sex with this partner, did you know his HIV status?" Being under the influence of drugs during sex with most recent sex partner was defined as "The last time you had sex with this partner, were you high or buzzed on [alcohol, drugs, both alcohol and drugs, neither]?" For the analyses subset, we selected only those NHBS and WHBS MSM who reported that their most recent male sex partner was a casual partner.

\section{Analyses}

We only analyzed data from persons who reported being currently of male gender and having sex with another man during the past 12 months. Descriptive analyses were conducted for venue attendance frequency by WHBS participant characteristics and venue type. A multivariate logistic regression analysis was performed to determine which participant characteristics were independently associated with never attending a venue during the past 12 months. All participant characteristics were retained in this model. 
Using the combined data from NHBS-MSM and WHBS, we developed a second multivariate logistic regression model to compare participant characteristics and behaviors between NHBS and WHBS MSM. To further describe the sexual risk behavior of WHBS MSM, a third multivariate logistic regression model (using only WHBS data) was developed to identify those factors independently associated with meeting the most recent casual male sex partner online. All participant characteristics and behaviors were retained in the second and third models.

Results for multivariate logistic regression models are presented as Wald chi-square p-values and/or adjusted odds ratios (aOR) with 95\% confidence intervals (95\% CI). All models controlled for MSA of residence, but the data are not presented. Statistical significance was determined at $\mathrm{p}<0.05$. All analyses were performed using SAS version 9.1 (SAS Institute, Cary, NC).

\section{RESULTS}

\section{Recruitment and Eligibility}

From April to August 2007, 21.9 million WHBS recruitment banner ad impressions resulted in 44,801 $(0.20 \%)$ persons entering the WHBS website and beginning the eligibility screener. Of those, 12,392 (28\%) currently resided in one of the 5 WHBS MSAs, of whom 11,979 $(97 \%)$ were age $\geq 18$ years and male at birth. A total of $6,261(52 \%)$ eligible persons completed the interview. For these analyses, 1,081 were excluded because they did not report being male gender or having sex with another man during the past 12 months.

From November 2003 to April 2005, 11,337 persons were approached for NHBS-MSM participation in the 5 MSAs included in this analysis. Eligibility interviews were completed with 9,055 persons $(80 \%)$, of whom $7,978(88 \%)$ were eligible for participation. Of these, 6,276 (79\%) agreed to participate and 5,667 (90\%) completed the interview. For these analyses, 951 were excluded because they did not report being male gender or having sex with another man during the past 12 months.

\section{Venue Attendance}

A total of 5,024 WHBS MSM were included in the venue attendance analysis; 156 persons were excluded from this analysis because of missing venue attendance, race/ethnicity or sexual identity information. Ninety-five percent of WHBS MSM reported attending any venue during the past 12 months, and 75\% reported attending at least weekly (Table 1). Venue attendance in the past 12 months was less likely among those who were non-Hispanic black (compared with other race/ethnicities, $\mathrm{aOR}=0.5, \quad \mathrm{CI}=0.4-0.8, \mathrm{p}<0.0001)$, under the age of 21 (compared with 21 or older, $\mathrm{aOR}=0.5$, $\mathrm{CI}=0.4-0.7, \mathrm{p}<0.0001)$, not college educated $(\mathrm{aOR}=0.7$, $\mathrm{CI}=0.5-0.9, \mathrm{p}<0.001$ ), bisexual or heterosexual identifying (compared to homosexual, $\mathrm{aOR}=0.3, \mathrm{CI}=0.3-0.4, \mathrm{p}<0.0001$ ), and of unknown HIV serostatus (compared with known HIV serostatus, $\quad \mathrm{aOR}=0.3, \quad \mathrm{CI}=0.3-0.4, \quad \mathrm{p}<0.0001)$. Venue attendance in the past 12 months was more likely among MSM who were HIV-positive compared with MSM who were not HIV-positive $(\mathrm{aOR}=4.3, \mathrm{CI}=1.3-13.7, \mathrm{p}=0.01)$.
Restaurants, bars, and retail businesses were the 3 most commonly reported venues attended, and more than a third of WHBS MSM attended these locations at least weekly (Table 2). Forty-six percent (2120/4584) of NHBS MSM reported using gay or bisexual chat rooms during the past 12 months, and 64\% (1354/2120) of those MSM reported using them at least weekly.

\section{Study Comparison}

A total of 2,607 NHBS MSM and 2,617 WHBS MSM were included in the comparison analyses. These totals were the result of excluding 2,106 NHBS MSM and 2,407 WHBS MSM because their most recent male sex partner was not a casual sex partner (more than $90 \%$ of the exclusions) or there was missing information for race/ethnicity, sexual identity or risk behavior variables (less than $10 \%$ of the exclusions). MSM differed significantly between the two studies for all participant characteristics and behaviors examined, except for sexual identity (Table 3). Compared to NHBS MSM, a larger proportion of WHBS MSM were non-Hispanic white, under the age of 31 , college educated, reported unknown HIV serostatus, engaged in unprotected anal intercourse with their most recent casual male partner, and first met that partner online. Compared to NHBS MSM, a smaller proportion of WHBS MSM were under the influence of drugs during last sex with a casual male partner and did not know the HIV serostatus of that partner.

\section{WHBS MSM Meeting Partners Online}

Those WHBS MSM who met their most recent casual male sex partner online were more likely than those who met their partner elsewhere to be 21 years or older $(\mathrm{aOR}=1.3$, $\mathrm{CI}=1.0-1.7, \mathrm{p}=0.02$ ), know their own HIV serostatus $(\mathrm{aOR}=1.3, \mathrm{CI}=1.0-1.6, \mathrm{p}=0.02)$, know the HIV serostatus of that sex partner $(\mathrm{aOR}=1.5, \mathrm{CI}=1.3-1.8, \mathrm{p}<0.0001)$, and engage in unprotected anal intercourse with that partner $(\mathrm{aOR}=1.2, \mathrm{CI}=1.0-1.5, \mathrm{p}=0.02)$.

\section{DISCUSSION}

The vast majority of MSM participants in WHBS had, at least once in the past year, attended one of the types of physical venue where NHBS recruitment takes place and three-quarters had attended at least weekly. Bars were one of the types of venues most commonly attended by WHBS MSM and were the venues where more than $1 / 3$ of NHBS MSM were recruited [5]. Attendance at venues was less likely for some subgroups of WHBS MSM: those who were black, young, not college educated, and bisexual or heterosexual identifying. One hypothesis to explain these findings would be that these subgroups may be reluctant to be indirectly identified as gay or bisexual through their attendance at venues frequented by MSM. Younger MSM may also not be of legal age to attend some of these venues (e.g., bars or clubs). WHBS MSM with unknown serostatus were also less likely to attend public venues. MSM who do not access these venues may be less likely to encounter HIV testing services offered through community outreach or community health centers in/near the venues [20] or could have a general lack of access to or knowledge about HIV testing and prevention services for MSM in their community [21]. 
Table 1. Frequency of Venue Attendance ${ }^{a}$ by MSM Participant Characteristics During the Past 12 Months, Web-Based HIV Behavioral Surveillance System (2007)

\begin{tabular}{|c|c|c|c|c|c|c|c|}
\hline & Total No. & \multicolumn{2}{|c|}{ Did Not Attend } & \multicolumn{2}{|c|}{ Attended Monthly or Less } & \multicolumn{2}{|c|}{ Attended Weekly or More } \\
\hline Total & 5024 & 244 & (5) & 1003 & (20) & 3777 & (75) \\
\hline \multicolumn{8}{|l|}{ Race/Ethnicity } \\
\hline White, not Hispanic & 3244 & 134 & (4) & 643 & $(20)$ & 2467 & $(76)$ \\
\hline Black, not Hispanic & 372 & 41 & (11) & 88 & (24) & 243 & $(65)$ \\
\hline Asian or Pacific Islander & 181 & 9 & (5) & 40 & $(22)$ & 132 & (73) \\
\hline Multiracial or other race/ethnicity & 292 & 14 & (5) & 49 & (17) & 229 & (78) \\
\hline \multicolumn{8}{|l|}{ Age } \\
\hline $18-20$ & 947 & 111 & (12) & 219 & $(23)$ & 617 & $(65)$ \\
\hline \multicolumn{8}{|l|}{ College Education } \\
\hline No & 917 & 94 & $(10)$ & 206 & $(22)$ & 617 & $(67)$ \\
\hline Yes & 4107 & 150 & (4) & 797 & (19) & 3160 & (77) \\
\hline \multicolumn{8}{|l|}{ Sexual Identity } \\
\hline Homosexual & 4335 & 149 & (3) & 842 & (19) & 3344 & $(77)$ \\
\hline Bisexual & 661 & 88 & (13) & 156 & $(24)$ & 417 & $(63)$ \\
\hline Heterosexual & 28 & 7 & (25) & 5 & $(18)$ & 16 & (57) \\
\hline \multicolumn{8}{|l|}{ HIV Serostatus ${ }^{b}$} \\
\hline Positive & 369 & 3 & $(1)$ & 60 & $(16)$ & 306 & $(83)$ \\
\hline Negative & 3715 & 121 & (3) & 712 & (19) & 2882 & $(78)$ \\
\hline
\end{tabular}

MSM $=$ men who have sex with men

${ }^{a}$ Attended a public venue where gay or bisexual men can be found.

${ }^{\mathrm{b}}$ Self-reported results of last HIV test; untested/unknown includes those never HIV tested or did not know the results of their last test.

Table 2. Frequency of Venue Attendance ${ }^{a}$ by Venue Type During the Past 12 Months Among MSM Participants, Web-Based HIV Behavioral Surveillance System (2007)

\begin{tabular}{|c|c|c|c|c|c|c|}
\hline & \multicolumn{2}{|c|}{ Did Not Attend } & \multicolumn{2}{|c|}{ Attended Monthly or Less } & \multicolumn{2}{|c|}{ Attended Weekly or More } \\
\hline Café, cybercafe or restaurant & 1371 & $(28)$ & 1450 & $(30)$ & 2012 & $(42)$ \\
\hline Bar & 1451 & (29) & 1804 & (36) & 1747 & $(35)$ \\
\hline Retail business & 1562 & (33) & 1554 & $(32)$ & 1671 & $(35)$ \\
\hline Dance Club & 1822 & (36) & 2088 & $(42)$ & 1087 & $(22)$ \\
\hline Sex establishment & 2079 & (43) & 2621 & (55) & 99 & (2) \\
\hline House party & 2223 & $(45)$ & 2193 & $(44)$ & 551 & $(11)$ \\
\hline Fitness club or gynasium & 2589 & $(53)$ & 479 & $(10)$ & 1844 & $(38)$ \\
\hline Social organization & 2858 & $(58)$ & 1387 & $(28)$ & 669 & (14) \\
\hline Street location & 2849 & $(59)$ & 982 & $(20)$ & 965 & $(20)$ \\
\hline
\end{tabular}

MSM = men who have sex with men.

${ }^{a}$ Attended a venue where gay or bisexual men can be found. Proportions denote the attendance frequencies among those who responded to the question regarding that venue type (some data is missing). 
Table 3. Characteristics and Behaviors of MSM Participants in the National HIV Behavioral Surveillance System (2003-2005) and the Web-Based HIV Behavioral Surveillance System (2007)

\begin{tabular}{|c|c|c|c|c|c|}
\hline & \multicolumn{2}{|c|}{$\operatorname{NHBS}(n=2607)$} & \multicolumn{2}{|c|}{ WHBS $(n=2617)$} & Wald Chi-Square p-Value \\
\hline White, not Hispanic & 1254 & $(48)$ & 1685 & $(64)$ & \\
\hline Black, not Hispanic & 435 & (17) & 215 & (8) & \\
\hline Asian or Pacific Islander & 190 & (7) & 100 & (4) & \\
\hline Multiracial or other race/ethnicity & 189 & (7) & 156 & $(6)$ & \\
\hline \multicolumn{5}{|l|}{ Age } & $\mathrm{p}<0.0001$ \\
\hline $18-20$ & 104 & (4) & 466 & $(18)$ & \\
\hline College Education & 2024 & $(78)$ & 2185 & $(83)$ & $\mathrm{p}<0.0001$ \\
\hline \multicolumn{5}{|l|}{ Sexual Identity } & $\mathrm{p}=0.87$ \\
\hline Homosexual & 2180 & $(84)$ & 2181 & $(83)$ & \\
\hline Bisexual & 400 & $(15)$ & 413 & $(16)$ & \\
\hline Heterosexual & 27 & (1) & 23 & (1) & \\
\hline \multicolumn{5}{|l|}{ HIV Serostatus ${ }^{b}$} & $\mathrm{p}<0.0001$ \\
\hline Positive & 361 & (14) & 230 & (9) & \\
\hline Negative & 1969 & (76) & 1843 & (70) & \\
\hline
\end{tabular}

MSM = men who have sex with men. NHBS = National HIV Behavioral Surveillance System. WHBS = Web-based HIV Behavioral Surveillance System.

${ }^{a}$ Wald Chi-square p-value from multivariate logistic regression model; model also included metropolitan statistical area of residence (data not shown).

${ }^{\mathrm{b}}$ Self-reported results of last HIV test; untested/unknown includes those never HIV tested or did not know the results of their last test.

There were also significant differences in the characteristics of participants in each study. Compared with NHBS MSM, WHBS MSM were more likely to be nonHispanic white, young, college-educated, and not know their HIV serostatus. Some of these differences (younger and unknown serostatus) may be explainable by lower venue attendance for these subgroups and others may be reflective of the internet-using MSM population in general, which has been reported by previous researchers to be mainly collegeeducated and white $[8,14,22]$.

Even accounting for these differences, MSM participants of WHBS were more likely than NHBS MSM to have engaged in unprotected anal intercourse with their most recent casual male partner but were also more likely to report that they knew their partner's HIV serostatus. WHBS MSM were also more likely to have met their partner online. One possible explanation of these findings is that MSM who use the internet for purposes of finding sex partners may seek partners of concordant HIV serostatus by examining selfreported serostatus in member profiles and may negotiate unprotected anal intercourse with seroconcordant partners $[18,23,24]$. The present WHBS analysis may provide some additional corroborating evidence for this hypothesis. When we examined the factors associated with meeting partners online among WHBS MSM, we found that they were more likely than those meeting partners elsewhere to have engaged in unprotected anal intercourse, know their own HIV serostatus, and reported knowing the HIV serostatus of their partner. Additional exploration of these issues for MSM who meet their partners online is warranted.

There are several limitations to our findings. First, these findings may not be generalizable to all MSM in the 5 participating MSAs. NHBS-MSM involved systematic sampling at venues where MSM congregate and WHBS used a convenience sampling method (banner ads) that resulted in low response rates and potential selection bias. We don't 
believe that the sampling or recruitment methods would have resulted in any systematic bias, but there is no way to confirm this through our existing data. The way that people utilize the internet for social networking has also been changing in the years since WHBS [25], which may reduce the applicability of these findings to any future web-based sampling method for MSM. Second, venue attendance was only measured for general types of venues and did not include specific names of venues, which limited specificity when drawing conclusions about whether WHBS MSM may have attended the exact venues used for NHBS-MSM sampling. Third, the differences between NHBS-MSM and WHBS questionnaires necessitated using only a subset of data for comparison analyses between the two studies. The subset represented approximately half of the total data available from each study and demographic characteristics significantly differed between those included in the subset and those who were not (data not presented): results of the comparison analyses may have differed if it had been feasible to include data from all participants. Fourth, although our results suggest that the sample of MSM obtained through WHBS methods differs from the NHBS sample, we do not know which sampling method captures a more representative sample of MSM or whether the samples may be complementary. We are also unable to determine whether the same individuals may have participated in both NHBS-MSM and WHBS. Fifth, we could only compare risk behaviors with the most recent casual male sex partner because the same data are not available in both NHBS-MSM and WHBS for main partners or for all partners during the past 12 months. Sixth, the serostatus of the participant and the most recent partner was not verified through HIV testing and self-reported measures of this type may be an inaccurate assessment of true HIV serostatus. Finally, asking for selfreports of sensitive information (e.g., sexual behavior, HIV serostatus) may result in underestimation of the prevalence of those behaviors. This may be particularly true with interviewer-administered surveys in the case of NHBS-MSM compared to self-administered surveys in the case of WHBS. The difference in survey administration method may partially explain the higher rates of unprotected anal intercourse reported by WHBS MSM, but other potentially sensitive information, such as drug use, were reported less frequently by WHBS MSM.

\section{CONCLUSION}

Though most groups of MSM recruited through WHBS methods should be reachable through NHBS-MSM sampling methods, web-based methods appear to be a useful means to increase access to some subgroups of MSM who don't attend venues used for NHBS-MSM sampling. If the intent is to better reach these subgroups, future online research will need to explore refinements to the WHBS sampling and recruitment methods. Compared to NHBS-MSM, WHBS also produced a sample of MSM who may be a higher risk of HIV infection due to higher rates of unprotected anal intercourse with casual male sex partners but these MSM may also be attempting to mitigate this risk through knowledge of their partner's HIV serostatus. Additional exploration of these issues for MSM who seek sex partners online would be useful for development of HIV prevention interventions.

\section{Human Participant Protection}

The WHBS pilot project was research conducted in compliance with applicable federal regulations governing protection of human subjects and was reviewed and approved by all local relevant human subjects review boards and the CDC institutional review board (CDC protocol 4481 and 4719). CDC determined that NHBS-MSM was public health surveillance and not a research activity. Local human subjects protection review was conducted according to policies of the institutions conducting NHBS-MSM.

\section{Web-based HIV Behavioral Surveillance Study Group}

Colin Flynn and Frangiscos Sifakis, Baltimore, MD; Eric Rubinstein and Gabriella Garcia, Boston, MA; Richard Yeager and Douglas Shehan, Dallas, TX; Trista Bingham and Alexander Carruth, Los Angeles, CA; Christopher Murrill and Sabina Hirshfield, New York City, NY; H. Fisher Raymond and Greg Rebchook, San Francisco, CA; Matthew Amsden, Reveal Communications; Paul Young and Benjamin Neal, NOVA Research Company.

\section{ACKNOWLEDGEMENT}

Declared none.

\section{CONFLICT OF INTEREST}

The authors confirm that this article content has no conflicts of interest.

\section{REFERENCES}

[1] Hall HI, Song R, Rhodes P et al. Estimation of HIV incidence in the United States. JAMA 2008; 300: 520-9.

[2] Sullivan PS, Hamouda O, Delpech V et al. Reemergence of the HIV epidemic among men who have sex with men in North America, Western Europe, and Australia, 1996-2005. Ann Epidemiol 2009; 19: 423-31.

[3] Centers for Disease Control and Prevention. HIV Prevention Strategic Plan Through 2005. 2001. Atlanta, Georgia, US Department of Health and Human Services, CDC. Available at: http://www.cdc.gov/hiv/resources/reports/psp/pdf/psp.pdf [Accessed 10 July 2012].

[4] Gallagher KM, Sullivan PS, Lansky A, Onorato IM. Behavioral surveillance among people at risk for HIV infection in the U.S.: the National HIV Behavioral Surveillance System. Public Health Rep 2007; 122(Suppl 1): 32-8.

[5] Centers for Disease Control and Prevention. Human immunodeficiency virus (HIV) risk, prevention, and testing behaviors--United States, National HIV Behavioral Surveillance System: men who have sex with men, November 2003-April 2005. MMWR Surveill Summ 2006; 55:1-16.

[6] MacKellar DA, Gallagher KM, Finlayson T, Sanchez T, Lansky A, Sullivan PS. Surveillance of HIV risk and prevention behaviors of men who have sex with men--a national application of venuebased, time-space sampling. Public Health Rep 2007; 122(Suppl 1): $39-47$.

[7] Ross MW, Tikkanen R, Mansson SA. Differences between Internet samples and conventional samples of men who have sex with men: implications for research and HIV interventions. Soc Sci Med 2000; 51: 749-58.

[8] Benotsch EG, Kalichman S, Cage M. Men who have met sex partners via the Internet: prevalence, predictors, and implications for HIV prevention. Arch Sex Behav 2002; 31: 177-83.

[9] Hirshfield S, Remien RH, Humberstone M, Walavalkar I, Chiasson MA. Substance use and high-risk sex among men who have sex with men: a national online study in the USA. AIDS Care 2004; 16:1036-47.

[10] Liau A, Millett G, Marks G. Meta-analytic examination of online sex-seeking and sexual risk behavior among men who have sex with men. Sex Transm Dis 2006; 33: 576-84. 
[11] Klausner JD, Wolf W, Fischer-Ponce L, Zolt I, Katz MH. Tracing a syphilis outbreak through cyberspace. JAMA 2000; 284: 447-9.

[12] Tashima KT, Alt EN, Harwell JI, Fiebich-Perez DK, Flanigan TP. Internet sex-seeking leads to acute HIV infection: a report of two cases. Int J STD AIDS 2003; 14: 285-6.

[13] Centers for Disease Control and Prevention. Internet use and early syphilis infection among men who have sex with men--San Francisco, California, 1999-2003. MMWR Morb Mortal Wkly Rep 2003; 52: 1229-32.

[14] Hirshfield S, Remien RH, Humberstone M, Walavalkar I, Chiasson MA. Substance use and high-risk sex among men who have sex with men: a national online study in the USA. AIDS Care 2004; 16: 1036-47.

[15] McFarlane M, Bull SS, Rietmeijer CA. The Internet as a newly emerging risk environment for sexually transmitted diseases. JAMA 2000; 284: 443-6.

[16] Hidaka Y, Ichikawa S, Koyano J et al. Substance use and sexual behaviours of Japanese men who have sex with men: a nationwide internet survey conducted in Japan. BMC Public Health 2006; 6: 239.

[17] Elford J, Bolding G, Davis M, Sherr L, Hart G. Web-based behavioral surveillance among men who have sex with men: a comparison of online and offline samples in London, UK. J Acquir Immune Defic Syndr 2004; 35: 421-6.

[18] Carballo-Dieguez A, Miner M, Dolezal C, Rosser BR, Jacoby S. Sexual negotiation, HIV-status disclosure, and sexual risk behavior among Latino men who use the internet to seek sex with other men. Arch Sex Behav 2006; 35: 473-81.
[19] Sanchez T, Smith A, Denson D, DiNenno E, Lansky A. Developing a Web-based HIV Behavioral Surveillance Pilot Project among Men Who Have Sex with Men. Open AIDS J 2012; 6: $\mathrm{xx}-\mathrm{xx}$.

[20] Bowles KE, Clark HA, Tai E et al. Implementing rapid HIV testing in outreach and community settings: results from an advancing HIV prevention demonstration project conducted in seven U.S. cities. Public Health Rep 2008; 123(Suppl 3): 78-85.

[21] Mills TC, Stall R, Pollack L et al. Health-related characteristics of men who have sex with men: a comparison of those living in "gay ghettos" with those living elsewhere. Am J Public Health 2001; 91 : 980-3.

[22] Bull SS, Lloyd L, Rietmeijer C, McFarlane M. Recruitment and retention of an online sample for an HIV prevention intervention targeting men who have sex with men: the Smart Sex Quest Project. AIDS Care 2004; 16: 931-43.

[23] McKirnan D, Houston E, Tolou-Shams M. Is the Web the culprit? Cognitive escape and Internet sexual risk among gay and bisexual men. AIDS Behav 2007; 11: 151-60.

[24] Davis M, Hart G, Bolding G, Sherr L, Elford J. Sex and the Internet: gay men, risk reduction and serostatus. Cult Health Sex 2006; 8: 161-74.

[25] Lenhart, A, Purcell, K, Smith, A, Zickuhr, K. Social Media and Young Adults. Pew Internet \& American Life Project. 3 Feb 2010. Pew Center. Available at: http://www.pewinternet.org/Reports/20 10/Social-Media-and-Young-Adults/Part-3/2-Adults-and-socialnetworks.aspx [Accessed: 10 July 2011].

(C) Sanchez et al.; Licensee Bentham Open.

This is an open access article licensed under the terms of the Creative Commons Attribution Non-Commercial License (http:/creativecommons.org/licenses/by-nc/ $3.0 /$ ) which permits unrestricted, non-commercial use, distribution and reproduction in any medium, provided the work is properly cited. 